\title{
Quasipinning and selection rules for excitations in atoms and molecules
}

\author{
Carlos L. Benavides-Riveros ${ }^{1,2,3, *}$ and Michael Springborg ${ }^{3,4}$ \\ ${ }^{1}$ Departamento de Física Teórica, Universidad de Zaragoza, 50009 Zaragoza, Spain \\ ${ }^{2}$ Instituto de Biocomputación y Física de Sistemas Complejos, 50018 Zaragoza, Spain \\ ${ }^{3}$ Physikalische und Theoretische Chemie, Universität des Saarlandes, 66123 Saarbrücken, Germany \\ ${ }^{4}$ School of Materials Science and Engineering, Tianjin University, Tianjin 300072, People's Republic of China
}

(Received 1 April 2015; published 16 July 2015)

\begin{abstract}
Postulated by Pauli to explain the electronic structure of atoms and molecules, the exclusion principle establishes an upper bound of 1 for fermionic natural occupation numbers $\left\{n_{i}\right\}$. A recent analysis of the pure $N$-representability problem provides a wide set of inequalities for the $\left\{n_{i}\right\}$, leading to constraints on these numbers. This has a strong potential impact on reduced density matrix functional theory as we know it. In this work we continue our study of the nature of these inequalities for some atomic and molecular systems. The results indicate that (quasi)saturation of some of them leads to selection rules for the dominant configurations in configuration interaction expansions, in favorable cases providing means for significantly reducing their computational requirements.
\end{abstract}

DOI: 10.1103/PhysRevA.92.012512

PACS number(s): 31.15.V-, 03.67.-a, 05.30.Fk

\section{INTRODUCTION}

Fermionic natural occupation numbers (arranged in the customary decreasing order $n_{i} \geqslant n_{i+1}$ ) fulfill the constraint $n_{i} \leqslant 1$, allowing no more than one electron in each quantum state. This condition, formulated by Coleman [1], is necessary and sufficient for a one-body reduced density matrix (1-RDM) to be the contraction of an ensemble $N$-body density matrix, provided that $\sum_{i} n_{i}=N$.

In a seminal work, Borland and Dennis [2] observed that for the rank 6 approximation of a pure-state $N=3$ system, belonging to the Hilbert space $\wedge^{3} \mathcal{H}_{6}$, the occupation numbers satisfy the following additional conditions: $n_{1}+n_{6}=n_{2}+$ $n_{5}=n_{3}+n_{4}=1$ and $n_{4} \leqslant n_{5}+n_{6}$. The set of equalities allows exactly one electron in the natural orbitals $r$ and $7-r$. The analysis by Klyachko and coworkers $[3,4]$ of the pure $N$-representability problem for the 1-RDM establishes a systematical approach, generalizing this type of constraint. For a pure quantum system of $N$ electrons arranged in $m$ spin orbitals, the occupation numbers satisfy a set of linear inequalities, known as generalized Pauli constraints (GPCs),

$$
D_{N, m}^{\mu}(\boldsymbol{n})=\kappa_{0}^{\mu}+\kappa_{1}^{\mu} n_{1}+\cdots+\kappa_{m}^{\mu} n_{m} \geqslant 0,
$$

with $\boldsymbol{n}:=\left(n_{1}, \ldots, n_{m}\right)$, the coefficients $\kappa_{j}^{\mu} \in \mathbb{Z}$, and $\mu=$ $1,2, \ldots, r_{N, m}$. These conditions define a convex polytope of allowed states in $\mathbb{R}^{m}$. When one of the GPCs is completely saturated [i.e., the equality holds in Eq. (1)], the system is said to be pinned, and it lies on one of the facets of the polytope.

The nature of those conditions has been explored till now in a few systems: a model of three spinless fermions confined to a one-dimensional harmonic potential [5], the lithium isoelectronic series [6], and ground and excited states of some three- and four-electron molecules with the rank being equal to twice the number of electrons [7]. For reasons that remain mysterious, for all these systems some inequalities are (quite often) nearly saturated; that is, in equations like (1), equality almost holds [8]. This is the so-called quasipinning

\footnotetext{
*carlosbe@unizar.es
}

phenomenon, originally proposed by Schilling, Gross, and Christandl [5].

The GPCs force a promissory rethinking of RDM functional theory, with possibly revolutionary consequences [9]. Also, violation of the GPCs has recently been identified as an encoder of the openness of a quantum system [10].

Since the dimension of the Hilbert space in the configuration interaction $(\mathrm{CI})$ method grows binomially with the number of electrons and of spin orbitals in the system, the method easily becomes very demanding numerically. Moreover, the CI expansion typically contains a great number of configurations that are superfluous (with very small expansion coefficients) for computing molecular electronic properties. Several approaches have been devised for selecting the most effective configurations in CI expansions [11,12]. Quasipinning offers another alley towards this end.

Let us consider one of the conditions of Eq. (1), $\mu$, for which pinning

$$
D_{N, m}^{\mu}(\boldsymbol{n})=0
$$

holds. An important superselection rule emerges for pinned wave functions [13]. In fact, given a pinned system satisfying equality (2), the corresponding wave function is an eigenfunction of a certain operator with eigenvalue 0 . As discussed in this paper, pinning enables the wave function to be described by an ansatz based on this selection rule, reducing the number of Slater determinants in the CI expansion. Recently, the stability of this selection rule (the potential loss of information when assuming pinning instead of quasipinning) has been measured for systems with nondegenerated natural orbitals which are close to the boundary of the polytope [14].

Here we examine the connection among pinning, quasipinning, and the excitation structure of the CI wave function in more detail. We identify those configurations that are negligible when imposing pinning on the wave function and study the issue of the robustness of quasipinning with increasing rank.

The paper is organized as follows. Section II elucidates the superselection rule for pinned systems. Section III is of 
a mathematical nature, as well. It is shown that there is still new wine in the old Borland-Dennis bottles: we prove, for not very strongly correlated systems, that the spin-compensated open-shell system $\wedge^{3} \mathcal{H}_{6}$ is always pinned to the boundary of the polytope described by the Borland-Dennis conditions. We then unveil a new regime for spin-compensated, strongly correlated systems, and finally, we briefly discuss the relation of GPCs to the linear equalities for reduced density matrices analyzed by Davidson and coworkers over many years $[15,16]$.

In Secs. IV and V we present results of numerical investigations for some atomic and molecular models: a lithium atom with broken spherical symmetry and the three-electron molecule $\mathrm{He}_{2}^{+}$, respectively. In Sec. VI we explore the connections among quasipinning, pinning, and the excitation structure of the CI wave function for three-electron systems. In the following section we discuss four-electron systems. Finally, in the last section (Sec. VIII) we summarize our conclusions. Throughout the paper we employ Hartree's atomic units (a.u.).

\section{SUPERSELECTION RULES}

In the full CI picture, the wave function in a given one-electron basis is expressed as a linear combination of all possible Slater determinants,

$$
|\Psi\rangle=\sum_{K} c_{K}|\mathbf{K}\rangle
$$

with $|\mathbf{K}\rangle$ denoting a determinant. Whenever we write expressions of this type in this paper, they are eigenfunctions of the spin operator $\mathbf{S}_{z}$, belonging to the same eigenvalue. In general, they will not be eigenfunctions of $\mathbf{S}^{2}$, so a spin adaptation is needed [17].

A one-body density matrix is compatible with the pure-state density matrix $|\Psi\rangle\langle\Psi|$ whenever its spectrum satisfies a set of linear inequalities of type (1). For pinned systems, such that condition (2) holds, the corresponding wave function belongs to the 0 -eigenspace of the operator

$$
\mathbf{D}_{N, m}^{\mu}=\kappa_{0}^{\mu} \mathbf{1}+\kappa_{1}^{\mu} a_{1}^{\dagger} a_{1}+\ldots+\kappa_{m}^{\mu} a_{m}^{\dagger} a_{m},
$$

where $a_{i}^{\dagger}$ and $a_{i}$ are the fermionic creation and annihilation operators of the state $i$. By using the expression of the wave function in the full CI picture, this condition can be recast into a superselection rule for the Slater determinants that appear in the CI decomposition. Given a pinned system that satisfies equality (2), each Slater determinant appearing in expansion (3) must be an eigenfunction of $\mathbf{D}_{N, m}^{\mu}$ with an eigenvalue equal to 0 . The superfluous or ineffective configurations are thus identified by means of the following criterion $[13,18]$ :

$$
\text { if } \quad \mathbf{D}_{N, m}^{\mu}|\boldsymbol{K}\rangle \neq 0, \text { then } \quad c_{K}=0 .
$$

The latter statement, for nondegenerate occupation numbers, follows from a relatively well-known result in symplectic geometry, whose proof can be traced back to the eighties [19]. The degenerate case needs a different kind of proof, which is forthcoming [20]. It immediately demonstrates that the (quasi)pinning phenomenon allows one to drastically reduce the number of Slater determinants in CI expansions.

The criterion becomes even more strict when more than one pinning constraint is satisfied. Were, for a given set of constraints $\left\{\mu_{1}, \mu_{2}, \ldots, \mu_{r}\right\}$, the corresponding GPCs to saturate, the ineffective configurations would satisfy simultaneously the selection rules,

$$
\text { if } \mathbf{D}_{N, m}^{\mu_{1}} \mathbf{D}_{N, m}^{\mu_{2}} \ldots \mathbf{D}_{N, m}^{\mu_{r}}|\mathbf{K}\rangle \neq 0, \quad \text { then } \quad c_{K}=0 .
$$

Note that the order of the operators $\mathbf{D}_{N, m}^{\mu}, \mathbf{D}_{N, m}^{v}$ is irrelevant, since they commute. For such selection rules, the occupation numbers satisfy the following set of simultaneous equations:

$$
D_{N, m}^{\mu_{1}}(\boldsymbol{n})=D_{N, m}^{\mu_{2}}(\boldsymbol{n})=\cdots=D_{N, m}^{\mu_{r}}(\boldsymbol{n})=0 .
$$

For instance, the Borland-Dennis state $\wedge^{3} \mathcal{H}_{6}$ satisfies

$$
\begin{aligned}
& \left(\mathbf{1}-a_{1}^{\dagger} a_{1}-a_{6}^{\dagger} a_{6}\right)|\Psi\rangle=0, \\
& \left(\mathbf{1}-a_{2}^{\dagger} a_{2}-a_{5}^{\dagger} a_{5}\right)|\Psi\rangle=0, \\
& \left(\mathbf{1}-a_{3}^{\dagger} a_{3}-a_{4}^{\dagger} a_{4}\right)|\Psi\rangle=0 .
\end{aligned}
$$

In general, it is not possible to saturate any arbitrary set of GPCs simultaneously, but as we discuss later certain sets are compatible with the rank of approximation when they are pinned.

In the remaining sections of this paper, among other things, we explore (in)effective configurations when a certain number of pinning conditions are imposed. We mainly deal with threeelectron systems, with Hilbert space $\wedge^{3} \mathcal{H}_{m}, m \geqslant 6$.

\section{EXACT PINNING IN SPIN-COMPENSATED CONFIGURATIONS FOR $\wedge{ }^{3} \mathcal{H}_{6}$}

For the rank 6 approximation for three-electron systems it is known [2] that the natural occupation numbers satisfy the constraints $n_{r}+n_{7-r}=1(r=1,2,3)$ and

$$
2-n_{1}-n_{2}-n_{4} \geqslant 0,
$$

where the numbers $\left\{n_{i}\right\}$ are arranged in the customary decreasing order $n_{i} \geqslant n_{i+1}$ and fulfill the Pauli condition $n_{1} \leqslant 1$. Inequality (4) together with the decreasing ordering rule defines a polytope (Fig. 1). Clearly, the smallest possible value for the first three occupation numbers, and the largest for the last three, is 0.5 .

The conditions $n_{r}+n_{7-r}=1$ imply that in the natural orbital basis, namely, $\left\{\alpha_{i}\right\}_{i=1}^{6}$, every Slater determinant is composed of three natural orbitals $\left|\alpha_{i} \alpha_{j} \alpha_{k}\right\rangle$, each one belonging to one of three different sets, say,

$$
\alpha_{i} \in\left\{\alpha_{1}, \alpha_{6}\right\}, \quad \alpha_{j} \in\left\{\alpha_{2}, \alpha_{5}\right\}, \quad \text { and } \quad \alpha_{k} \in\left\{\alpha_{3}, \alpha_{4}\right\} .
$$

This results in eight possible configurations:

$$
\begin{array}{llll}
\left|\alpha_{1} \alpha_{2} \alpha_{3}\right\rangle, & \left|\alpha_{1} \alpha_{2} \alpha_{4}\right\rangle, & \left|\alpha_{1} \alpha_{3} \alpha_{5}\right\rangle, & \left|\alpha_{1} \alpha_{4} \alpha_{5}\right\rangle, \\
\left|\alpha_{2} \alpha_{3} \alpha_{6}\right\rangle, & \left|\alpha_{2} \alpha_{4} \alpha_{6}\right\rangle, & \left|\alpha_{3} \alpha_{5} \alpha_{6}\right\rangle, & \left|\alpha_{4} \alpha_{5} \alpha_{6}\right\rangle .
\end{array}
$$

A spin-compensated configuration consists of three spin orbitals whose spin points down, and another three whose spin points up. Such a configuration is in general favorable for the energy in comparison with other types of arrangements [6]. The $1-R D M$ (a $6 \times 6$ matrix) is the direct sum of two $(3 \times 3)$ matrices, one related to the spin up and the other related to the spin down:

$$
\rho_{1}=\rho_{1}^{\uparrow} \oplus \rho_{1}^{\downarrow} .
$$




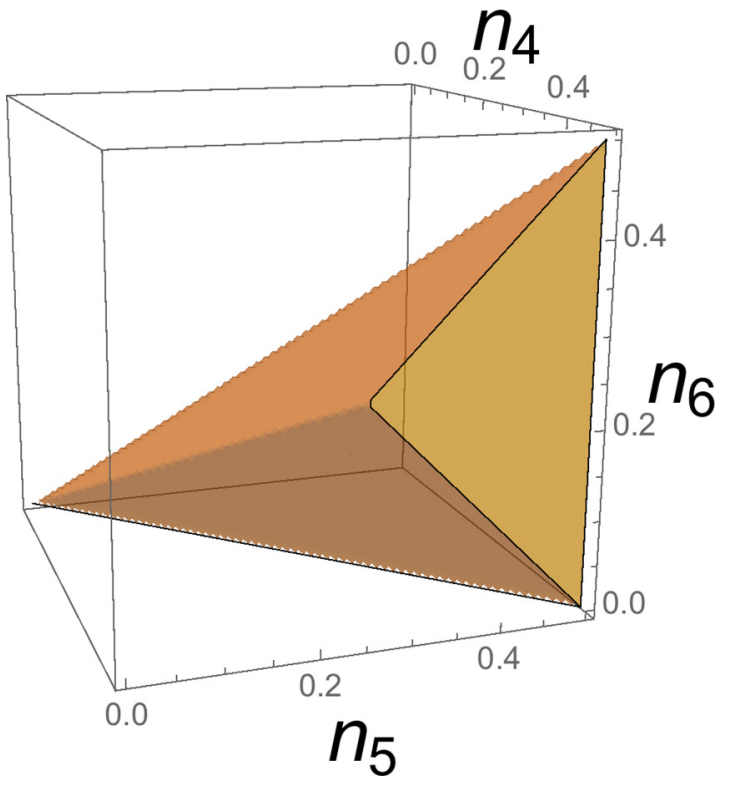

FIG. 1. (Color online) Polytope defined by the expression $n_{4} \leqslant$ $n_{5}+n_{6}$, subject to the condition $0 \leqslant n_{6} \leqslant n_{5} \leqslant n_{4} \leqslant 0.5$. The saturation condition $n_{5}+n_{6}=n_{4}$ is satisfied by the points on one of the faces of the polytope, whereas on the edges $n_{5}+n_{6}=0.5$ we have $n_{4}=0.5$ and for $n_{4}=n_{5}$ we have $n_{6}=0$. The single determinant state is placed in the lower left corner $n_{i}=0$ of the polytope.

The wave function is an eigenstate of the total spin operator $\mathbf{S}_{z}$ (and of $\mathbf{S}^{2}$ in the spin-restricted case). Therefore, each acceptable Slater determinant will contain two spin orbitals pointing up (for instance) and one pointing down. It follows that the trace of one of those matrices will be equal to 1 , while the sum of the diagonal elements of the other one will be equal to 2. Say,

$$
\operatorname{Tr} \rho_{1}^{\uparrow}=2 \text { and } \quad \operatorname{Tr} \rho_{1}^{\downarrow}=1 .
$$

For not very strongly correlated systems, two of the first three occupation numbers belong to the matrix whose trace is equal to 2 . Hence, we have the two conditions $n_{i}+n_{j}+n_{x}=$ 2 and $n_{k}+n_{y}+n_{z}=1$, where $i, j, k \in\{1,2,3\}$ and $x, y, z \in$ $\{4,5,6\}$. For a given $i$ and $j$ there are three possible values of $x$ and therefore there are, in principle, nine possible solutions:

$$
\begin{array}{lll}
n_{1}+n_{2}+n_{x}=2 & \text { and } & n_{3}+n_{y}+n_{z}=1, \\
n_{1}+n_{3}+n_{x}=2 & \text { and } & n_{2}+n_{y}+n_{z}=1, \\
n_{2}+n_{3}+n_{x}=2 & \text { and } & n_{1}+n_{y}+n_{z}=1 .
\end{array}
$$

However, we may easily dismiss all but one of them. For instance, the case

$$
n_{2}+n_{3}+n_{6}=2 \text { and } n_{1}+n_{4}+n_{5}=1
$$

is impossible: using $n_{1}=1-n_{6}$, one obtains $-n_{6}+n_{4}+$ $n_{5}=0$. This would imply that $n_{4}=n_{6}-n_{5} \leqslant 0$, which is out of the question. Also, for

$$
n_{1}+n_{3}+n_{5}=2 \text { and } n_{2}+n_{4}+n_{6}=1,
$$

using that $n_{2}=1-n_{5}$, one obtains $-n_{5}+n_{4}+n_{6}=0$, which would imply that $n_{4}=n_{5}-n_{6}<n_{5}$. Other cases are easily seen to give rise to, at most, rank 4 or 5 for the wave function, except for

$$
n_{1}+n_{2}+n_{4}=2 \text { and } n_{3}+n_{5}+n_{6}=1,
$$

which saturates the representability condition, (4). Therefore, for not very strongly correlated systems the spin-compensated wave function of $\wedge^{3} \mathcal{H}_{6}$ lies on one of the facets of the Borland-Dennis-Klyachko polytope. This is in agreement with the numerical results obtained previously [6,7]. A similar phenomenon can be observed in the Hubbard model for three fermions on three lattice sites with periodic conditions [21].

The wave function for this configuration for $\wedge^{3} \mathcal{H}_{6}$ in the basis of natural orbitals can now be written in terms of the 1-RDM matrix,

$$
|\Psi\rangle_{3,6}=c_{123}\left|\alpha_{1} \alpha_{2} \alpha_{3}\right\rangle+c_{145}\left|\alpha_{1} \alpha_{4} \alpha_{5}\right\rangle+c_{246}\left|\alpha_{2} \alpha_{4} \alpha_{6}\right\rangle,
$$

with the proviso that $\left|c_{123}\right| \geqslant\left|c_{145}\right| \geqslant\left|c_{246}\right|$. It is now patent that $|\Psi\rangle$ can be elegantly rewritten as

$$
\sqrt{n_{3}}\left|\alpha_{1} \alpha_{2} \alpha_{3}\right\rangle+\sqrt{n_{5}}\left|\alpha_{1} \alpha_{4} \alpha_{5}\right\rangle+\sqrt{n_{6}}\left|\alpha_{2} \alpha_{4} \alpha_{6}\right\rangle,
$$

in analogy to the Löwdin-Shull functional for the two-electron case [22]. Note that, just like in the Löwdin-Shull functional, only doubly excited configurations are permitted here [23]. (We understand excitations with respect to the "best density" Slater determinant, in the sense of [24]).

The pinned configuration

$$
\left(n_{1}, n_{2}, n_{3}\right)=\left(\frac{3}{4}, \frac{3}{4}, \frac{1}{2}\right)
$$

is far from the "Hartree-Fock" $(1,1,1)$ state. Now, a little surprise awaits us: for spin-compensated, very strongly correlated systems it is possible to show, by the same method as above, the identity

$$
\begin{aligned}
& n_{1}+n_{2}+n_{3}=2 ; \\
& \quad \text { equivalently, } n_{4}+n_{5}+n_{6}=1 .
\end{aligned}
$$

In terms of $\rho_{1}$ the wave function then reads

$$
|\Psi\rangle=\sqrt{n_{4}}\left|\alpha_{1} \alpha_{2} \alpha_{4}\right\rangle+\sqrt{n_{5}}\left|\alpha_{1} \alpha_{3} \alpha_{5}\right\rangle+\sqrt{n_{6}}\left|\alpha_{2} \alpha_{3} \alpha_{6}\right\rangle,
$$

living in the 0 -eigenspace of the operator

$$
\mathbf{2}-a_{1}^{\dagger} a_{1}-a_{2}^{\dagger} a_{2}-a_{3}^{\dagger} a_{3} .
$$

We note that overlap of those wave functions with the $\left|\alpha_{1} \alpha_{2} \alpha_{3}\right\rangle$ state is 0 . For the case $n_{4}=n_{5}=n_{6}=1 / 3$ this was noted by Kutzelnigg and Smith [24]. The Borland-Dennis-Klyachko constraint becomes, in this case,

$$
2-\left(n_{1}+n_{2}+n_{4}\right)=n_{3}-n_{4} \geqslant 0 .
$$

Therefore in this regime it is possible to determine $|\Psi\rangle$ from $\rho_{1}$ even without Klyachko pinning. The border between the two regimes is given by the degeneracy line $n_{3}=n_{4}=\frac{1}{2}$. Inequality (4) together with the pinning, (7), cut out a new facet on the polytope of allowed states (Fig. 2).

In summary, the Borland-Dennis-Klyachko polytope of states is still too large. In fact, the spin-compensated states lie either on the $n_{1}+n_{2}+n_{4}=2$ facet of the polytope (when closer to the single-determinant state) or on the plane $n_{4}+n_{5}+n_{6}=1$ (when farther from the single-determinant state). The edge $n_{3}=n_{4}$ is shared by these two planes. Since the exact expressions given above for the spin-compensated formulation of the system $\wedge^{3} \mathcal{H}_{6}$ lead to a diagonal 1-RDM, 


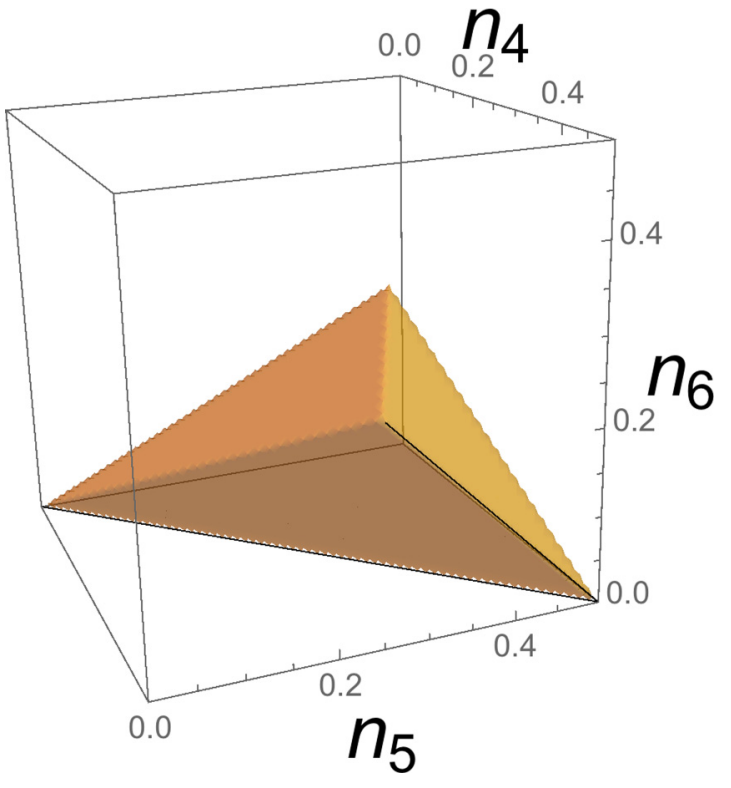

FIG. 2. (Color online) Polytope defined by the expression $n_{4} \leqslant$ $n_{5}+n_{6}$, subject to the condition $0 \leqslant n_{6} \leqslant n_{5} \leqslant n_{4} \leqslant 0.5$, plus the condition $n_{4}+n_{5}+n_{6} \leqslant 1$. The saturation condition $n_{4}+n_{5}+$ $n_{6}=1$ is satisfied by the points on the face of the polytope whose vertices are $\left(\frac{1}{2}, \frac{1}{2}, 0\right),\left(\frac{1}{2}, \frac{1}{4}, \frac{1}{4}\right)$, and $\left(\frac{1}{3}, \frac{1}{3}, \frac{1}{3}\right)$.

without any restriction on the amplitudes $c_{i j k}$ (provided, of course, that the orbitals are orthonormal), for such a simple system one does not need a previous CI calculation to compute the natural orbitals or the value of the ground-state energy [8].

A well-known extension of density functional theory is the pair-density functional theory, which is based on the diagonal elements of the 2-RDM [25]. It is known that the corresponding representability constraints are expressed in terms of the latter elements. For the system $\wedge^{3} \mathcal{H}_{6}$ there are, in total, 70 Slater hull inequalities, grouped into three permutation classes $[15,16]$. Since the 2-RDM is a positive semidefinite matrix its diagonal elements are never negative. The first class is thus given by 15 inequalities,

$$
\left\langle\hat{n}_{i} \hat{n}_{j}\right\rangle \geqslant 0, \quad 1 \leqslant i<j \leqslant 6,
$$

where $\hat{n}_{i}:=a_{i}^{\dagger} a_{i}$ is the occupation number operator; the second class contains 10 inequalities, namely,

$$
\left\langle\left(\hat{n}_{f}+\hat{n}_{g}+\hat{n}_{h}\right)^{2}+\left(\hat{n}_{i}+\hat{n}_{j}+\hat{n}_{k}\right)^{2}\right\rangle \geqslant 5,
$$

provided that $1 \leqslant f, g, h, i, j, k \leqslant 6$; and the last one is determined by 45 inequalities of the type

$$
\left\langle\left(\hat{n}_{f}+\hat{n}_{g}\right)\left(\hat{n}_{h}+\hat{n}_{i}\right)+\hat{n}_{f} \hat{n}_{g}+\hat{n}_{h} \hat{n}_{i}+\hat{n}_{j} \hat{n}_{k}\right\rangle \geqslant 1 .
$$

For the wave function, (5), the majority of these inequalities are pinned. Six inequalities of the first class are completely saturated, namely,

$$
\left\langle\hat{n}_{i} \hat{n}_{7-i}\right\rangle=0 \quad \text { and } \quad\left\langle\hat{n}_{3} \hat{n}_{5}\right\rangle=\left\langle\hat{n}_{3} \hat{n}_{6}\right\rangle=\left\langle\hat{n}_{5} \hat{n}_{6}\right\rangle=0 .
$$

For the second class [Eq. (8)] we have that all are pinned save

$$
\begin{aligned}
& \left\langle\left(\hat{n}_{1}+\hat{n}_{2}+\hat{n}_{3}\right)^{2}+\left(\hat{n}_{4}+\hat{n}_{5}+\hat{n}_{6}\right)^{2}\right\rangle=5+4 n_{3}>5, \\
& \left\langle\left(\hat{n}_{1}+\hat{n}_{4}+\hat{n}_{5}\right)^{2}+\left(\hat{n}_{2}+\hat{n}_{3}+\hat{n}_{6}\right)^{2}\right\rangle=5+4 n_{5}>5, \\
& \left\langle\left(\hat{n}_{2}+\hat{n}_{4}+\hat{n}_{6}\right)^{2}+\left(\hat{n}_{1}+\hat{n}_{3}+\hat{n}_{5}\right)^{2}\right\rangle=5+4 n_{6}>5 .
\end{aligned}
$$

Since the occupation numbers are arranged in decreasing order, the strongest nonpinned inequality is the first one. The number of linearly independent inequalities belonging to the third class [Eq. (9)] drops to only 9. For instance,

$$
\left\langle\left(\hat{n}_{1}+\hat{n}_{6}\right)\left(\hat{n}_{2}+\hat{n}_{3}\right)+\hat{n}_{1} \hat{n}_{6}+\hat{n}_{2} \hat{n}_{3}+\hat{n}_{4} \hat{n}_{5}\right\rangle=1+n_{3}>1 .
$$

In the end, we deal with 21 inequalities instead of 70 , a remarkable reduction. It is worth mentioning that the $N$ representability constraints for the ensemble 2-RDM contain as a subset all of these "classical" $N$-representability conditions $[26,27]$.

\section{NUMERICAL INVESTIGATIONS: LITHIUM WITH BROKEN SPHERICAL SYMMETRY}

In the previous paper [6] we obtained rank 6,7 , and 8 approximations for the lithium isoelectronic series by using a set of helium-like one-particle wave functions in addition to one hydrogen-like wave function. Guided by the classical work of Shull and Löwdin [22], for the former we employed the set of orthonormal spatial orbitals

$$
\delta_{n}(\alpha, \boldsymbol{r}):=D_{n} \sqrt{\frac{\alpha^{3}}{\pi}} L_{n-1}^{2}(2 \alpha r) e^{-\alpha r}, \quad n=1,2, \ldots,
$$

where $D_{n}^{-2}=\left(\begin{array}{c}n-1 \\ 2\end{array}\right)$, and we use the standard definition of the associated Laguerre polynomials $L_{n}^{\zeta}$ [28]. For the hydrogenlike function we used

$$
\psi(\beta, \boldsymbol{r})=\frac{1}{4} \sqrt{\frac{\beta^{5}}{6 \pi}} r e^{-\beta r / 2} .
$$

Applying a variational procedure for the state $\left|\delta_{1} \uparrow \delta_{1} \downarrow \psi \uparrow\right\rangle$ results in $\alpha=2.68$ and $\beta=1.27$, and the total energy associated with this Slater determinant becomes -7.4179 a.u., reasonably close to the Hartree-Fock energy, -7.4327 a.u.

Now we examine the GPC when the spherical symmetry of the central potential is broken by considering the following Hamiltonian:

$$
\begin{aligned}
H(Z, \gamma)= & \frac{1}{2} \sum_{i=1}^{3}\left|\boldsymbol{p}_{i}\right|^{2}-\sum_{i=1}^{3} \frac{Z}{\left|\boldsymbol{r}_{i}\right|}\left(1+\gamma \frac{x_{i}^{2}}{\left|\boldsymbol{r}_{i}\right|^{2}}\right) \\
& +\sum_{i<j}^{3} \frac{1}{\left|\boldsymbol{r}_{i}-\boldsymbol{r}_{j}\right|} .
\end{aligned}
$$

The case $H(3,0)$ is the Hamiltonian of lithium, whose accurate energy value is -7.47806 a.u.

A motivation behind this model is to lift constraints on the possible occupation numbers due to the spherical symmetry of the isolated $\mathrm{Li}$ atom. Lowering the symmetry makes the model more flexible and allows us to envisage more general cases. In addition, the model can serve to describe a $\mathrm{Li}$ atom embedded in some environment that does not provide covalent interactions with the Li atom. We have performed the calculations of this section by searching those values of $\alpha$ and $\beta$ for which the approximation to the ground state leads to the minimum energy with spin-compensated linear combinations of Slater determinants. Analytical expressions for the electron integrals were computed using Mathematica [29] and orthonormalized orbitals were obtained by the 
Gram-Schmidt procedure. Computations were performed with 36-decimal floating-point precision.

\section{A. Rank 6}

The spin-restricted rank 6 approximation for $H(3, \gamma)$ is always pinned to the boundary of the polytope, as we have shown in the general case in Sec. III. It is interesting to examine the spectral trajectory of the "best" spin-restricted state in $\wedge^{3} \mathcal{H}_{6}$ as a function of the parameter $\gamma$ by means of minimizing the $\mathrm{CI}$ states on the manifold $(\alpha, \beta)$. To this end we choose as a one-particle Hilbert space the set

$$
\left\{\delta_{1} \uparrow, \delta_{1} \downarrow, \psi \uparrow, \psi \downarrow, \delta_{2} \uparrow, \delta_{2} \downarrow\right\} .
$$

The Hilbert space factorizes then in the direct product of two spin-orbital sectors, $\wedge^{3} \mathcal{H}_{6} \rightarrow \mathcal{H}_{3} \otimes \wedge^{2} \mathcal{H}_{3}$. There are nine configurations in all, eight of which belong to the $j=\frac{1}{2}$ representation:

$$
\begin{array}{lll}
\left|\delta_{1} \uparrow \delta_{1} \downarrow \psi \uparrow\right\rangle, & \left|\delta_{1} \uparrow \delta_{1} \downarrow \delta_{2} \uparrow\right\rangle, & \left|\psi \uparrow \psi \downarrow \delta_{1} \uparrow\right\rangle, \quad\left|\psi \uparrow \psi \downarrow \delta_{2} \uparrow\right\rangle, \\
\left|\delta_{2} \uparrow \delta_{2} \downarrow \delta_{1} \uparrow\right\rangle, & \left|\delta_{2} \uparrow \delta_{2} \downarrow \psi \uparrow\right\rangle, & \left|\delta_{1} \uparrow \psi \downarrow \delta_{2} \uparrow\right\rangle-\left|\delta_{1} \downarrow \psi \uparrow \delta_{2} \uparrow\right\rangle, \\
\left|\delta_{1} \uparrow \psi \uparrow \delta_{2} \downarrow\right\rangle-\left|\delta_{1} \downarrow \psi \uparrow \delta_{2} \uparrow\right\rangle . &
\end{array}
$$

(The configuration $\quad\left|\delta_{1} \uparrow \psi \downarrow \delta_{2} \uparrow\right\rangle+\left|\delta_{1} \downarrow \psi \uparrow \delta_{2} \uparrow\right\rangle+$ $\left|\delta_{1} \uparrow \psi \uparrow \delta_{2} \downarrow\right\rangle$ belongs to the representation $j=\frac{3}{2}$ ).

In order to quantify the position of the set of occupation numbers on the boundary of the polytope, one may define $\xi^{2}:=\sum_{i=1}^{3}\left(1-n_{i}\right)^{2}$ as the euclidean distance between the spectrum of the state and the extreme point $(1,1,1)$ of the polytope, corresponding to the spectrum of a single determinant. Figure 3 shows $\xi$ for small values of $\gamma$ of the electronic Hamiltonian given in (10). For decreasing $\gamma$ the spectrum of the one-body density departs farther from that extremum. The kinematics, however, keeps the state pinned to the boundary of the Borland-Dennis-Klyahcko polytope, since its natural occupation numbers maintain the condition $1+n_{3}=n_{1}+n_{2}$

A similar behavior is observed for a unrestricted description using, for instance, the set

$$
\left\{\delta_{1} \uparrow, \delta_{1} \downarrow, \psi \uparrow, \delta_{2} \uparrow, \delta_{2} \downarrow, \delta_{3} \uparrow\right\}
$$

as the one-particle Hilbert space. However, the energy predicted by the latter configuration is slightly worse than the one predicted by the spin-restricted case.

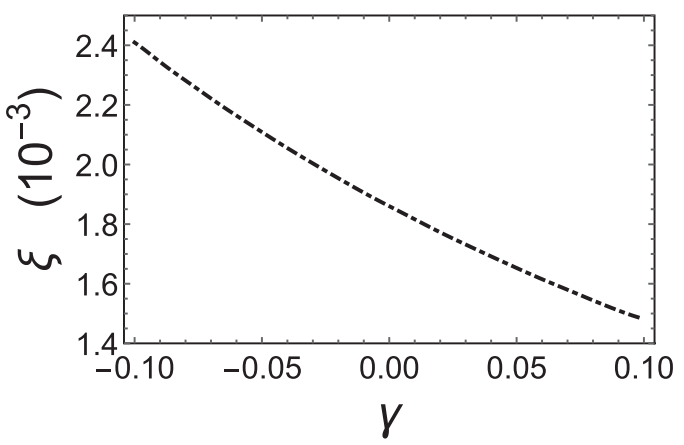

FIG. 3. The distance $\xi$ between the spectrum of the ground state and the extreme point of the polytope as a function of $\gamma \in[-0.1,0.1]$ for the spin-restricted rank 6 approximation to the Hamiltonian $H(3, \gamma)$ given by $(10)$.

\section{B. Rank 7}

There are four GPC for the three-electron system in a rank 7 configuration $\wedge^{3} \mathcal{H}_{7}$ :

$$
\begin{aligned}
& D_{3,7}^{1}=2-n_{1}-n_{2}-n_{4}-n_{7} \geqslant 0, \\
& D_{3,7}^{2}=2-n_{1}-n_{2}-n_{5}-n_{6} \geqslant 0, \\
& D_{3,7}^{3}=2-n_{2}-n_{3}-n_{4}-n_{5} \geqslant 0, \\
& D_{3,7}^{4}=2-n_{1}-n_{3}-n_{4}-n_{6} \geqslant 0 .
\end{aligned}
$$

For lithium-like atoms, calculations [6,7] had shown that the first of these four inequalities is completely saturated.

For the rank 7 approximation to the Hamiltonian, (10), we choose

$$
\left\{\delta_{1} \uparrow, \delta_{1} \downarrow, \psi \uparrow, \delta_{2} \uparrow, \delta_{2} \downarrow, \delta_{3} \uparrow, \delta_{3} \downarrow\right\}
$$

as the one-particle Hilbert space. Other types of configurations are possible too, but they lead to higher values for the groundstate energy. There are 18 configurations in total, but only 14 of them belong in the $j=\frac{1}{2}$ representation. For any $\gamma$, the occupation numbers satisfy

$$
n_{1}+n_{2}+n_{4}+n_{7}=2 \text { and } n_{3}+n_{5}+n_{6}=1,
$$

implying that the first GPC of (11) is completely saturated. The Hilbert space of this system then splits into the direct product of two spin-orbital sectors, $\wedge^{3} \mathcal{H}_{7} \rightarrow \mathcal{H}_{3} \otimes \wedge^{2} \mathcal{H}_{4}$.

Also, the following interesting system was analyzed in [13]. The first excited state of beryllium, with $\operatorname{spin}\left(\mathbf{S}, \mathbf{S}_{z}\right)=(1,1)$, fills the lowest three shells, $1 s, 2 s$, and $2 p$. In a reasonable approximation, the first natural orbital is completely occupied and the last two are empty (thus $n_{9}=n_{10}=0$ ). The seven remaining natural orbitals are organized in such a way that the first inequality in (11) is saturated too.

For lithium we found previously [6] that the GPC could be split into two groups differing in how close the equalities were obeyed; i.e., one may talk about two scales of quasipinning. Here we observe the same phenomenon. In fact, the value of the constraint $D_{3,7}^{2}$ is always below $1.3717 \times 10^{-5}$, taking its maximum for $\gamma=0.01$ (i.e., practically at the "physical point"), as indicated in Fig. 4.

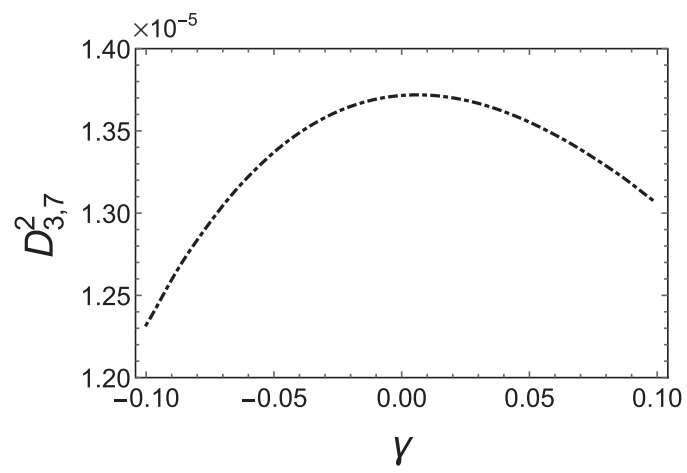

FIG. 4. Second GPC $D_{3,7}^{2}$ for the ground state of the Hamiltonian $H(3, \gamma)$ in a rank 7 approximation as a function of $\gamma \in[-0.1,0.1]$. For $\gamma=0.01$, the constraint reaches its maximum value (namely, $\left.1.3717 \times 10^{-5}\right)$. 


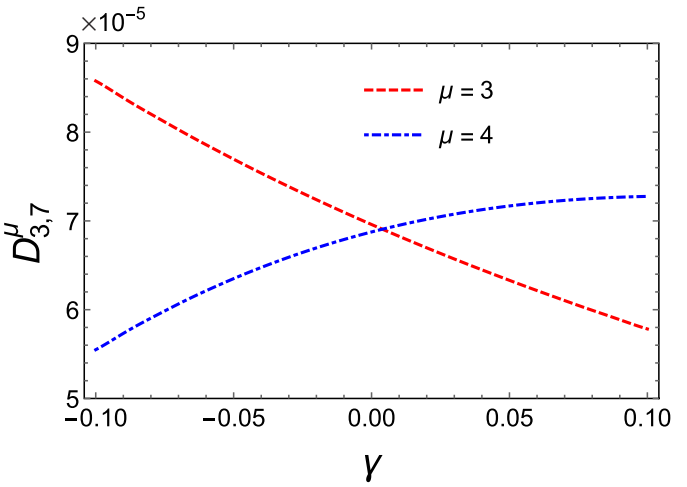

FIG. 5. (Color online) Third and fourth GPCs for the ground state of the Hamiltonian $H(3, \gamma)$ in a rank 7 approximation as a function of $\gamma \in[-0.1,0.1]$.

On the other hand, the remaining two GPCs, $D_{3,7}^{3}$ and $D_{3,7}^{4}$, take values around $7 \times 10^{-5}$. As shown in Fig. 5, $D_{3,7}^{3}$ decreases when the value of $\gamma$ grows, while the last one increases when $\gamma$ increases. Note the crossover of two constraints also close by $\gamma=0$.

\section{On the energies}

Finally, Fig. 6 depicts the ground-state energy predicted by our model for the spin-restricted version of the rank 6 approximation as a function of $\gamma$. For $H(3,0)$ the ground-state energy is -7.4311 a.u. For rank 7 and $\gamma=0$, the calculated energy for this model is equal to -7.4458 a.u., lower than the Hartree-Fock energy for lithium. Remarkably, the rank 8 approximation for this model gives -7.4548 a.u. for the ground-state energy of lithium, which represents more than $50 \%$ of its correlation energy [6].

\section{THE MOLECULAR SYSTEM He $\mathrm{H}_{2}^{+}$}

In this section we study the behavior of the occupation numbers of helium's molecular ion $\mathrm{He}_{2}^{+}$. The goal is to explore the GPCs along the dissociation path of this threeelectron system, whose symmetry is lower than spherical, identifying those almost saturated. The Hartree-Fock energy for this system is -4.9 a.u. [30], with a $6-31 \mathrm{G}$ basis set. The equilibrium bond length is 2.08 a.u. [30]. The computed value for the ground-state energy is approximately -4.99 a.u. $[30,31]$. Therefore the correlation energy is equal to $90 \mathrm{mHa}$. We have approximated the atomic orbitals by employing a 6-31G basis set [32]. We here report our results for (rank 6, 7, and 8) CI approximations for this diatomic ion.

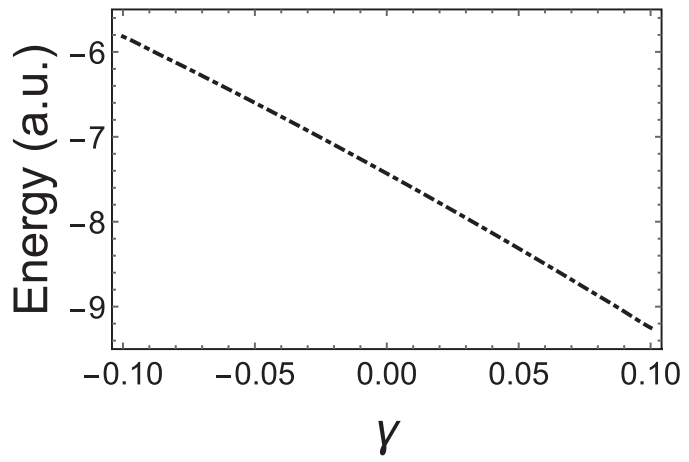

FIG. 6. Ground-state energy for the spin-restricted rank 6 approximation to the Hamiltonian $H(3, \gamma)$ given by (10) as a function of $\gamma \in[-0.1,0.1]$.

\section{A. GPC for the $\mathrm{He}_{2}^{+}$ground state}

For a dimer with atomic charges $Z$ the energy is given by the expression

$$
\begin{aligned}
& -\left.\int\left(\frac{1}{2} \nabla_{\boldsymbol{r}}^{2}+\sum_{\mu \in\{A, B\}} \frac{Z}{\left|\boldsymbol{r}-\boldsymbol{R}_{\mu}\right|}\right) \rho_{1}\left(\boldsymbol{x}, \boldsymbol{x}^{\prime}\right)\right|_{\boldsymbol{x}=\boldsymbol{x}^{\prime}} d \boldsymbol{x} \\
& +\int \frac{\rho_{2}\left(\boldsymbol{x}_{1}, \boldsymbol{x}_{2}\right)}{\left|\boldsymbol{r}_{1}-\boldsymbol{r}_{2}\right|} d \boldsymbol{x}_{1} d \boldsymbol{x}_{2}+\frac{Z^{2}}{|\boldsymbol{R}|} .
\end{aligned}
$$

The two atoms are located at $\boldsymbol{R}_{A}$ and $\boldsymbol{R}_{B}$ and separated by $\boldsymbol{R}:=\boldsymbol{R}_{A}-\boldsymbol{R}_{B}$. The standard quantum-chemical notation, $\boldsymbol{x}:=(\boldsymbol{r}, \zeta)$, with $\varsigma \in\{\uparrow, \downarrow\}$ is employed. The molecular orbitals are constructed as linear combinations of the atomic $1 s$ and $2 s$ orbitals, which are in turn solutions of the Hartree-Fock equations. In the rest of this subsection, standard notation for the bonding (gerade) and antibonding (ungerade) molecular orbitals is used. The ground-state configuration of $\mathrm{He}_{2}^{+}$is classified as ${ }^{2} \Sigma_{u}$ and the starting configuration is a single Slater determinant, $\left|1 \sigma_{g}^{\uparrow} 1 \sigma_{g}^{\downarrow} 1 \sigma_{u}^{\uparrow}\right\rangle$.

Table I lists the results for the energy and for the natural orbital occupancy numbers from rank 6 up to rank 8 approximations for the ground state of $\mathrm{He}_{2}^{+}$. The rank 6 approximation is obtained through a spin-compensated configuration,

$$
\left\{1 \sigma_{g}^{\uparrow}, 1 \sigma_{g}^{\downarrow}, 1 \sigma_{u}^{\uparrow}, 1 \sigma_{u}^{\downarrow}, 2 \sigma_{g}^{\uparrow}, 2 \sigma_{g}^{\downarrow}\right\} .
$$

Higher-rank configurations are obtained by successively adding the orbitals $\left\{2 \sigma_{u}^{\uparrow}, 2 \sigma_{u}^{\downarrow}\right\}$.

A number of findings can now be identified.

(a) For rank 6, the spin-compensated configuration gives the Borland-Dennis-Klyachko saturation condition $1+n_{3}=$ $n_{1}+n_{2}$.

(b) For rank 7, we obtain the following values for the GPC:

$$
\begin{array}{ll}
D_{3,7}^{1}=2.42 \times 10^{-5}, & D_{3,7}^{2}=0, \\
D_{3,7}^{3}=1.24 \times 10^{-3}, & D_{3,7}^{4}=1.39 \times 10^{-3} .
\end{array}
$$

TABLE I. Occupation numbers and energies for rank 6 to rank 8 for $\mathrm{He}_{2}^{+}$in its equilibrium geometry, employing 6-31G basis set.

\begin{tabular}{lccccccccc}
\hline \hline Rank & Energy & $n_{1}$ & $n_{2}$ & $n_{3}$ & $n_{4}\left(10^{-3}\right)$ & $n_{5}\left(10^{-3}\right)$ & $n_{6}\left(10^{-3}\right)$ & $n_{7}\left(10^{-3}\right)$ & $n_{8}\left(10^{-4}\right)$ \\
\hline 6 & -4.9125 & 0.9992 & 0.9949 & 0.9941 & 5.8086 & 5.0914 & 0.7172 & - \\
7 & -4.9194 & 0.9973 & 0.9941 & 0.9915 & 7.1019 & 5.8950 & 2.5530 & 1.3220 \\
8 & -4.9239 & 0.9968 & 0.9932 & 0.9901 & 8.4888 & 6.8304 & 3.0819 & 1.3665 & 0.1178 \\
\hline \hline
\end{tabular}


TABLE II. First 19 GPCs for the system $\wedge{ }^{3} \mathcal{H}_{8}$ and numerical values for $\mathrm{He}_{2}^{+}$at its equilibrium geometry.

\begin{tabular}{lc}
\hline \hline Generalized Pauli condition for $\wedge{ }^{3} \mathcal{H}_{8}$ & $\times 10^{3}$ \\
\hline $0 \leqslant D_{3,8}^{1}=2-\left(n_{1}+n_{2}+n_{4}+n_{7}\right)$ & 0.0570 \\
$0 \leqslant D_{3,8}^{2}=2-\left(n_{1}+n_{2}+n_{5}+n_{6}\right)$ & 0 \\
$0 \leqslant D_{3,8}^{3}=2-\left(n_{2}+n_{3}+n_{4}+n_{5}\right)$ & 1.2712 \\
$0 \leqslant D_{3,8}^{4}=2-\left(n_{1}+n_{3}+n_{4}+n_{6}\right)$ & 1.4854 \\
$0 \leqslant D_{3,8}^{5}=1-\left(n_{1}+n_{2}-n_{3}\right)$ & 0.0452 \\
$0 \leqslant D_{3,8}^{6}=1-\left(n_{2}+n_{5}-n_{7}\right)$ & 1.2594 \\
$0 \leqslant D_{3,8}^{7}=1-\left(n_{1}+n_{6}-n_{7}\right)$ & 1.4736 \\
$0 \leqslant D_{3,8}^{8}=1-\left(n_{2}+n_{4}-n_{6}\right)$ & 1.3164 \\
$0 \leqslant D_{3,8}^{9}=1-\left(n_{1}+n_{4}-n_{5}\right)$ & 1.5306 \\
$0 \leqslant D_{3,8}^{10}=1-\left(n_{3}+n_{4}-n_{7}\right)$ & 2.7449 \\
$0 \leqslant D_{3,8}^{11}=1-\left(n_{1}+n_{8}\right)$ & 3.1772 \\
$0 \leqslant D_{3,8}^{12}=-\left(n_{2}-n_{3}-n_{6}-n_{7}\right)$ & 1.3046 \\
$0 \leqslant D_{3,8}^{13}=-\left(n_{4}-n_{5}-n_{6}-n_{7}\right)$ & 2.7901 \\
$0 \leqslant D_{3,8}^{14}=-\left(n_{1}-n_{3}-n_{5}-n_{7}\right)$ & 1.5188 \\
$0 \leqslant D_{3,8}^{15}=2-\left(n_{2}+n_{3}+2 n_{4}-n_{5}-n_{7}+n_{8}\right)$ & 7.7980 \\
$0 \leqslant D_{3,8}^{16}=2-\left(n_{1}+n_{3}+2 n_{4}-n_{5}-n_{6}+n_{8}\right)$ & 5.9792 \\
$0 \leqslant D_{3,8}^{17}=2-\left(n_{1}+2 n_{2}-n_{3}+n_{4}-n_{5}+n_{8}\right)$ & 5.0983 \\
$0 \leqslant D_{3,8}^{18}=2-\left(n_{1}+2 n_{2}-n_{3}+n_{5}-n_{6}+n_{8}\right)$ & 3.0082 \\
$0 \leqslant D_{3,8}^{19}=-\left(n_{1}+n_{2}-2 n_{3}-n_{4}-n_{5}\right)$ & 5.4973 \\
\hline \hline
\end{tabular}

The constraint due to spin has "jumped," with respect to the lithium series!

(c) For the latter rank, two scales of quasipinning are clearly identified. Compared with the lithium-like atom, the first level of quasipinning is here more meaningful and probably more useful in order to reduce the number of Slater determinants, since the distance to the "Hartee-Fock" point is bigger, namely, $\xi=1.06 \times 10^{-2}$.

It is a fact of life that the number of GPCs grows very rapidly with rank. For rank 8 there are 31 inequalities [4]. They have been listed in a plain-text format [33]. Of these, 19 constraints are listed in Table II. The first four are equal to the Klyachko conditions for $\wedge^{3} \mathcal{H}_{7}$.

Several scales of quasipinning can be identified here, as well. Most important is the robustness of quasipinning. In particular, the quantity $D_{3,8}^{2}$, found to be exactly 0 in the previous rank, remains in a saturated regime. The first and fifth inequalities belong to a strongly quasipinned regime too. For the remaining inequalities we have

$$
\begin{aligned}
& D_{3,8}^{2} \leqslant D_{3,8}^{5} \leqslant D_{3,8}^{1} \leqslant D_{3,8}^{6} \leqslant D_{3,8}^{3} \\
& \leqslant D_{3,8}^{12} \leqslant D_{3,8}^{8} \leqslant D_{3,8}^{7} \leqslant D_{3,8}^{4} \leqslant \ldots
\end{aligned}
$$

\section{B. Occupation numbers and potential curves}

Potential energy curves for the three different ranks of the CI approximation for $\mathrm{He}_{2}^{+}$are presented in Fig. 7. At the equilibrium geometry, as also reported in Table I, a larger rank results in a lower ground-state energy. All approximations behave similarly around the equilibrium distance. At large interatomic distances, the value predicted by the rank 8 configuration is -4.845 a.u. which is to be compared with the total energy of the two separated compounds $\left(\mathrm{He}\right.$ and $\mathrm{He}^{+}$): -4.903 a.u. [34].

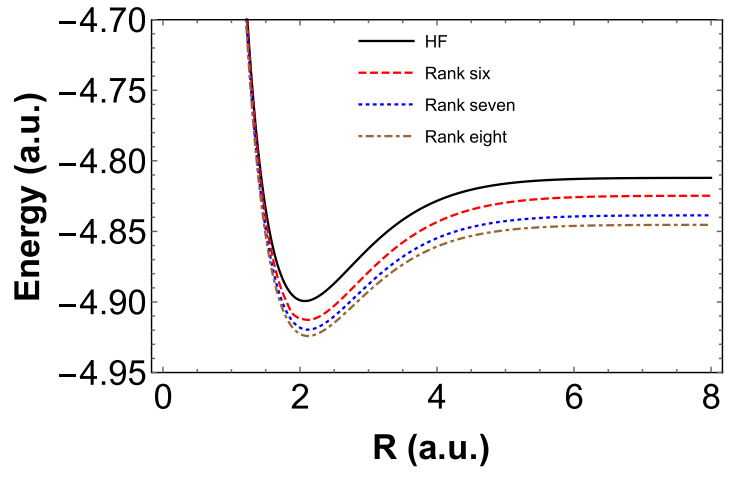

FIG. 7. (Color online) $\mathrm{He}_{2}^{+}$potential energy curves for the three ranks of CI approximation $\wedge^{3} \mathcal{H}_{m}, m \in\{3,6,7,8\}$ using 6-31G as a basis set.

Figure 8 displays rank 7 GPCs as functions of the interatomic distance in atomic units. There are again two scales of quasipinning. The first two GPCs remain in a strongly pinned regime, since for these, $D_{3,7}^{\mu}$ is very close to 0 . For those, we notice a sharp crossover at lengths shorter than that of equilibrium. In fact, one of them is always completely saturated: in the region $R<1.25$ a.u., i.e., $D_{3,7}^{1}=0$ is a very good approximation, whereas for $R>1.25$ a.u., $D_{3,7}^{2}=0$ is also very good. Unfortunately, we do not yet have a good description for this apparent quenching of degrees of freedom, which surely deserves further investigation.

For rank 8 , several scales of quasipinning can be observed for $\mathrm{He}_{2}^{+}$. Our main result is again the robustness of
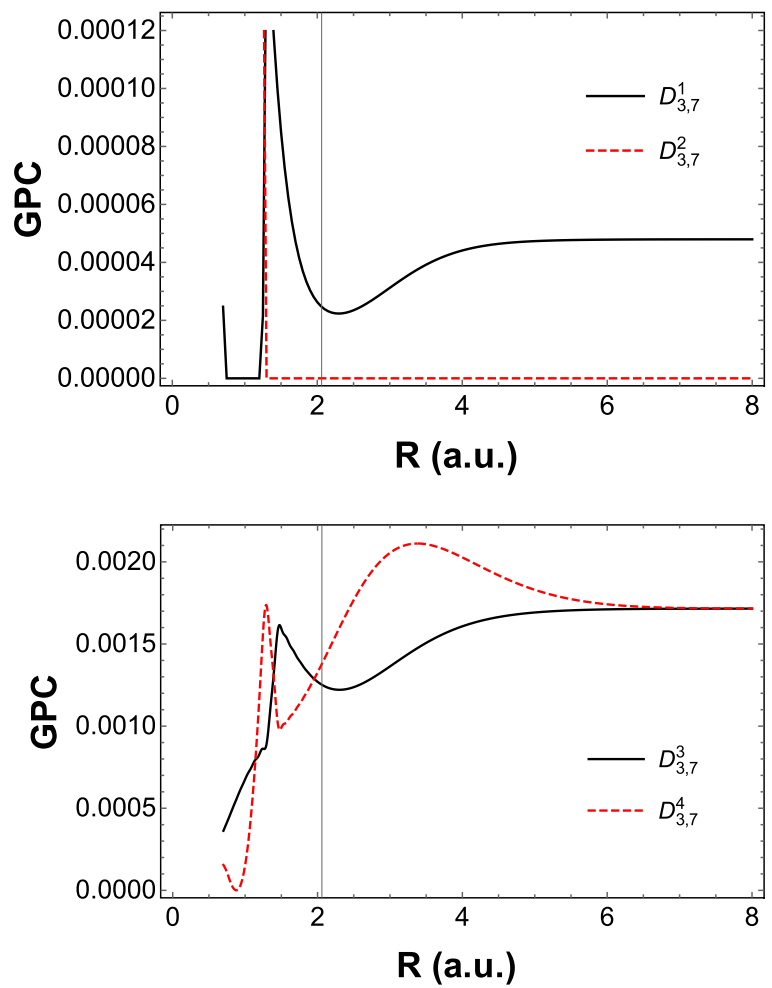

FIG. 8. (Color online) Rank 7 GPCs as functions of the interatomic distance in atomic units. Vertical lines mark the equilibrium bond lengths. 

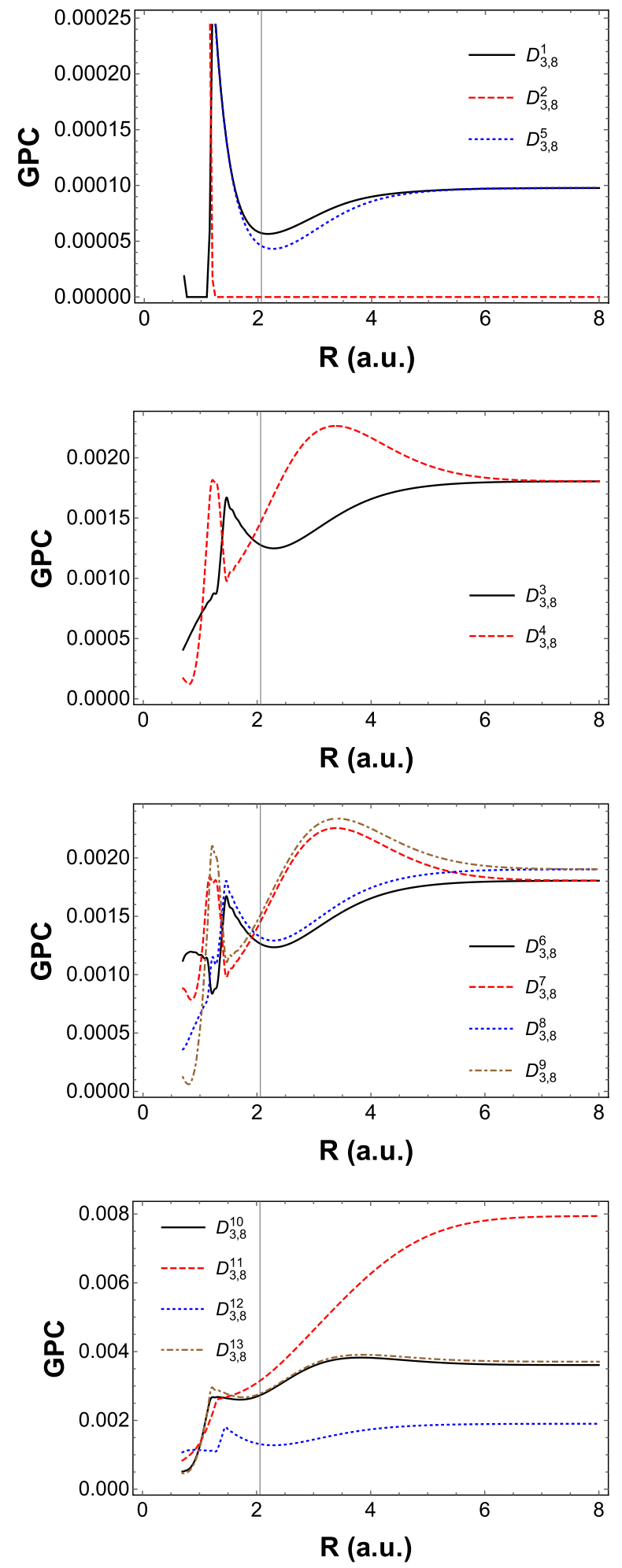

FIG. 9. (Color online) Rank 8 GPCs as functions of the interatomic distance. Vertical lines mark the equilibrium bond lengths.

quasipinning. In particular, we observe that the quantities $D_{3,8}^{1}$ and $D_{3,8}^{2}$, found to be exactly 0 for some bond-length regime at rank 7 , remain in a strongly saturated regime, as shown in Fig. 9. The Hilbert space of this system then splits into the direct product of two spin-orbital sectors, $\wedge^{3} \mathcal{H}_{8} \rightarrow \mathcal{H}_{4} \otimes \wedge^{2} \mathcal{H}_{4}$. Also, $D_{3,8}^{5}$ is found to be very close to 0 .

To a second quasipinning regime belong the quantities

$D_{3,8}^{3}, \quad D_{3,8}^{4}, \quad D_{3,8}^{6}, \quad D_{3,8}^{7}, \quad D_{3,8}^{8}, \quad D_{3,8}^{9}, \quad D_{3,8}^{12}, \quad D_{3,8}^{14}$.

As shown in Fig. 9, these GPCs behave roughly in the same way for increasing bond lengths. Their values tend asymptotically to approximately the same value for large interatomic distances.

Finally, a third quasipinning sector appears to be composed of $D_{3,8}^{10}, D_{3,8}^{13}, D_{3,8}^{15}, D_{3,8}^{18}, D_{3,8}^{19}$.

\section{QUASIPINNING AND EXCITATIONS}

From the seminal work by Löwdin and Shull it is known that the transformation to natural orbitals removes all single (S) excitations of the wave function of two-electron systems [22]. For the singlet state the general wave function can be written exactly as

$$
\left|\Psi\left(\boldsymbol{x}_{1}, \boldsymbol{x}_{2}\right)\right\rangle=\frac{1}{\sqrt{2}}\left(\uparrow_{1} \downarrow_{2}-\downarrow_{1} \uparrow_{2}\right) \sum_{i=1}^{\infty} c_{i}\left|\alpha_{i}\left(\boldsymbol{r}_{1}\right) \alpha_{i}\left(\boldsymbol{r}_{2}\right)\right\rangle .
$$

Again, we have used $\boldsymbol{x}:=(\boldsymbol{r}, \varsigma)$, with $\varsigma$ being the spin coordinates $\{\uparrow, \downarrow\}$. A similar expression can be found for the triplet state [35].

It is also remarkable that the wave function, (5), does not contain S or triple (T) excitations of the best single-determinant state $|0\rangle:=\left|\alpha_{1} \alpha_{2} \alpha_{3}\right\rangle$. The Slater determinants $\left|\alpha_{1} \alpha_{4} \alpha_{5}\right\rangle$ and $\left|\alpha_{2} \alpha_{4} \alpha_{6}\right\rangle$ correspond to double (D) excitations of this state.

Single excitations cannot be completely removed from the CI wave function of general many-electron systems when written in terms of natural orbitals. However, Mentel and coworkers [36] have recently shown that writing the wave function in the basis of natural orbitals leads to a sharp drop of the coefficients of Slater determinants containing just single excitations. For the $\mathrm{BH}$ molecule, the sum of squares of $\mathrm{CI}$ coefficients of singles falls from $1.5 \times 10^{-3}$ to $5.3 \times 10^{-6}$ when switching to the natural orbital basis. In this section and the next we argue that this phenomenon is a consequence of the near-saturation of some Klyachko selection rules on the occupation numbers.

\section{A. Selection rule for excitations in $\wedge^{3} \mathcal{H}_{6}$}

This case has been just discussed. Even if the number of basis spin orbitals pointing up is different from the number pointing down, an eventual saturation of condition (4) would lead to the situation summarized in Table III. A double excitation is also removed thereby.

TABLE III. Number of Slater determinants in the total and forcepinned CI expansions of the wave function for the system $\wedge{ }^{3} \mathcal{H}_{6}$.

\begin{tabular}{lccccc}
\hline \hline Condition & $|0\rangle$ & $\mathrm{S}$ & $\mathrm{D}$ & $\mathrm{T}$ & Total \\
\hline $\mathrm{CI}$ & 1 & 3 & 3 & 1 & 8 \\
$\mathbf{D}_{3,6}^{1}|\Psi\rangle=0$ & 1 & 0 & 2 & 0 & 3 \\
\hline \hline
\end{tabular}


TABLE IV. Number of Slater determinants in the total and forcepinned CI expansions of the wave function for the system $\wedge{ }^{3} \mathcal{H}_{7}$.

\begin{tabular}{lccccc}
\hline \hline Condition & $|0\rangle$ & $\mathrm{S}$ & $\mathrm{D}$ & $\mathrm{T}$ & Total \\
\hline $\mathbf{D}_{3,7}^{1}|\Psi\rangle_{3,7}=0$ & 1 & 6 & 9 & 2 & 18 \\
$\mathbf{D}_{3,7}^{2} \mathbf{D}_{3,7}^{1}|\tilde{\Psi}\rangle_{3,7}=0$ & 1 & 0 & 8 & 0 & 9 \\
\hline \hline
\end{tabular}

\section{B. Selection rules for excitations in $\wedge^{3} \mathcal{H}_{7}$}

The four Klyachko inequalities for the three-electron system in a rank 7 approximation $\wedge^{3} \mathcal{H}_{7}$ were given in Eq. (11). The corresponding operators are

$$
\begin{aligned}
& \mathbf{D}_{3,7}^{1}=\mathbf{2}-a_{1}^{\dagger} a_{1}-a_{2}^{\dagger} a_{2}-a_{4}^{\dagger} a_{4}-a_{7}^{\dagger} a_{7}, \\
& \mathbf{D}_{3,7}^{2}=\mathbf{2}-a_{1}^{\dagger} a_{1}-a_{2}^{\dagger} a_{2}-a_{5}^{\dagger} a_{5}-a_{6}^{\dagger} a_{6}, \\
& \mathbf{D}_{3,7}^{3}=\mathbf{2}-a_{2}^{\dagger} a_{2}-a_{3}^{\dagger} a_{3}-a_{4}^{\dagger} a_{4}-a_{5}^{\dagger} a_{5}, \\
& \mathbf{D}_{3,7}^{4}=\mathbf{2}-a_{1}^{\dagger} a_{1}-a_{3}^{\dagger} a_{3}-a_{4}^{\dagger} a_{4}-a_{6}^{\dagger} a_{6} .
\end{aligned}
$$

As discussed above, for the lithium isoelectronic series [6], for the system described by the Hamiltonian of Eq. (10) and for the first excited state of beryllium in a rank 10 approximation [13], the first of the four inequalities (11) is completely saturated. Accordingly, for all these systems, the exact wave function satisfies the condition

$$
\mathbf{D}_{3,7}^{1}|\Psi\rangle_{3,7}=0
$$

This implies that in the natural orbital basis, every Slater determinant is composed of three natural orbitals, two of them belonging to the set $\left\{\alpha_{1}, \alpha_{2}, \alpha_{4}, \alpha_{7}\right\}$ and one belonging to the set $\left\{\alpha_{3}, \alpha_{5}, \alpha_{6}\right\}$. Then the system $\wedge^{3} \mathcal{H}_{7}$ is reduced to $\mathcal{H}_{3} \otimes \wedge^{2} \mathcal{H}_{4}$, with, in total, 18 of these Slater determinants.

Imposing, in addition, saturation of the second inequality of (11), i.e., $\mathbf{D}_{3,7}^{2} \mathbf{D}_{3,7}^{1}|\tilde{\Psi}\rangle_{3,7}=0$, the singles and the triples are completely removed from the expression, as reported in Table IV. The corresponding wave function $|\tilde{\Psi}\rangle_{3,7}$ is written in terms of the inicial configuration $\left|\alpha_{1} \alpha_{2} \alpha_{3}\right\rangle$, plus the following eight configurations:

$$
\begin{array}{llll}
\left|\alpha_{1} \alpha_{4} \alpha_{5}\right\rangle, & \left|\alpha_{2} \alpha_{4} \alpha_{6}\right\rangle, & \left|\alpha_{1} \alpha_{5} \alpha_{7}\right\rangle, & \left|\alpha_{2} \alpha_{5} \alpha_{7}\right\rangle, \\
\left|\alpha_{1} \alpha_{4} \alpha_{6}\right\rangle, & \left|\alpha_{2} \alpha_{4} \alpha_{5}\right\rangle, & \left|\alpha_{1} \alpha_{6} \alpha_{7}\right\rangle, & \left|\alpha_{2} \alpha_{6} \alpha_{7}\right\rangle .
\end{array}
$$

\section{Selection rules for excitations in $\wedge^{3} \mathcal{H}_{8}$}

The empirical evidence discussed earlier shows that the inequalities for the following GPCs are almost or completely saturated:

$$
D_{3,8}^{1}, \quad D_{3,8}^{2}, \quad D_{3,8}^{5} \text {. }
$$

Imposing the saturation of the second and fifth constraints, say, the singles and the triples are removed completely, as reported in Table V. The corresponding wave function $|\tilde{\Psi}\rangle_{3,8}$ is written in terms of the nineconfigurations of the pinned rank 7 wave function, (13), plus the configurations

$$
\left|\alpha_{1} \alpha_{5} \alpha_{8}\right\rangle, \quad\left|\alpha_{2} \alpha_{6} \alpha_{8}\right\rangle, \quad\left|\alpha_{2} \alpha_{5} \alpha_{8}\right\rangle, \quad\left|\alpha_{1} \alpha_{6} \alpha_{8}\right\rangle
$$

TABLE V. Number of Slater determinants in the total and forcepinned CI expansions of the wave function for the system $\wedge^{3} \mathcal{H}_{8}$.

\begin{tabular}{lccccc}
\hline \hline Condition & $|0\rangle$ & $\mathrm{S}$ & $\mathrm{D}$ & $\mathrm{T}$ & Total \\
\hline $\mathbf{D}_{3,8}^{2}|\Psi\rangle_{3,8}=0$ & 1 & 7 & 13 & 3 & 24 \\
$\mathbf{D}_{3,8}^{5} \mathbf{D}_{3,8}^{2}|\tilde{\Psi}\rangle_{3,8}=0$ & 1 & 0 & 12 & 0 & 13 \\
\hline \hline
\end{tabular}

\section{D. $\mathrm{He}_{2}^{+}$: Electronic energy and pinning truncations}

An idea behind quasipinning is to approximate the wave function through a truncated expansion by using the selection rules that emerge after imposing pinning. Therefore, it is a relevant issue to examine how the electronic energy is affected as the number of configurations is reduced in the truncation. Here we explore the ground-state energy for the helium dimer $\mathrm{He}_{2}^{+}$for different pinned wave functions, compared with the energy predicted by the CI expansion within the same rank. (It must be said beforehand that, contrary to the case of lithiumlike systems, up to rank 8 , less than $30 \%$ of the absolute correlation energy is recovered. This is due partly to a less than optimal choice of the basis set and partly to the difficulty of capturing some aspects of correlation with such short basis sets).

Table VI lists the value of the correlation energy for (force-pinned and complete) wave functions for rank 6 up to rank 8 approximations for the ground state of $\mathrm{He}_{2}^{+}$. It is remarkable that the force-pinned wave function $|\tilde{\Psi}\rangle_{3,7}$ reconstructs $99.79 \%$ of the rank 7 correlation energy, employing just nine configurations. The CI rank 8 wave function contains 24 Slater determinants belonging to the Hilbert space $\wedge^{2} \mathcal{H}_{4} \otimes \mathcal{H}_{4}$. The correlation energy is $24.64 \mathrm{mHa}$. The pinned wave function $|\tilde{\Psi}\rangle_{3,8}$ reconstructs $99.51 \%$ of this available correlation energy, employing 13 Slater determinants.

Figure 10 shows the absolute value of the correlation energy along the dissociation path for CI rank 6 up to rank 8 expansions $\left(|\Psi\rangle_{3,6},|\Psi\rangle_{3,7},|\Psi\rangle_{3,8}\right)$ and for the pinned wave functions $|\tilde{\Psi}\rangle_{3,7}$ and $|\tilde{\Psi}\rangle_{3,8}$. It is also remarkable that the pinned rank 7 and rank 8 wave functions almost contain the complete correlation energy to the corresponding rank of approximation along the complete path, demonstrating the negligible role of the single and triple excitations. These results suggest that even though that saturation of one GPC notably reduces the number of Slater determinants, remarkably good values for the correlation energies are obtained.

TABLE VI. Ground-state correlation energies predicted for the complete and force-pinned CI wave functions for $\mathrm{He}_{2}^{+}$in rank 6 up to rank 8 approximations. Values are given in $\mathrm{mHa}$.

\begin{tabular}{lc}
\hline \hline Wave function & $\left|E-E_{\mathrm{HF}}\right|$ \\
\hline$|\Psi\rangle_{3,6}$ & 13.22 \\
$|\tilde{\Psi}\rangle_{3,7}$ & 20.12 \\
$|\Psi\rangle_{3,7}$ & 20.17 \\
$|\tilde{\Psi}\rangle_{3,8}$ & 24.56 \\
$|\Psi\rangle_{3,8}$ & 24.64 \\
\hline \hline
\end{tabular}




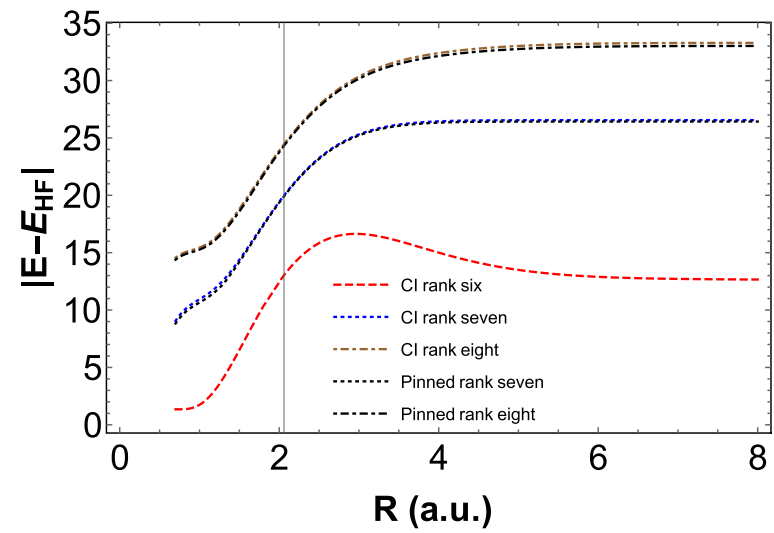

FIG. 10. (Color online) Absolute value of the correlation energy $\left|E-E_{\mathrm{HF}}\right|$ for $\mathrm{CI}$ expansion and pinned wave functions for rank 6 up to rank 8 approximations, along the dissociation path for $\mathrm{He}_{2}^{+}$. The pinned wave function $|\tilde{\Psi}\rangle_{3,6}$ is equivalent to the CI rank 6 expansion, so it is not included. Values are given in $\mathrm{mHa}$.

\section{ON FOUR-ELECTRON SYSTEMS}

For the case of a four-electron system with an eightdimensional one-electron Hilbert space, $\wedge^{4} \mathcal{H}_{8}$, there are, in total, 14 GPC. Derived initially by Klyachko [4], they read

$$
\begin{aligned}
D_{4,8}^{\mu} & :=\sum_{i=1}^{8} \kappa_{i}^{\mu} n_{i} \geqslant 0 \\
D_{4,8}^{7+\mu} & :=2-\sum_{i=1}^{8} \kappa_{9-i}^{\mu} n_{i} \geqslant 0,
\end{aligned}
$$

for $1 \leqslant \mu \leqslant 7$ and provided that $n_{1} \leqslant 1$. The coefficients $\kappa_{i}^{\mu}$ are listed in Table VII.

For quantum states with an even number of fermions, vanishing total spin, and time-reversal symmetry, Smith proved that a $1-\mathrm{RDM}$ is pure $N$-representable if and only if all its eigenvalues are doubly degenerated [37]. Therefore, for these systems, the occupation numbers obey

$$
n_{2 i-1}=n_{2 i}, \quad i=1,2, \ldots
$$

The double degeneracy of the occupation numbers forces the GPCs for the system $\wedge^{4} \mathcal{H}_{8}$ to reduce to the traditional Pauli exclusion principle [7]. Therefore, a state will be pinned only if it is pinned to the traditional Pauli conditions, which only

TABLE VII. Sets of coefficients for the GPC of (14) for the system $\wedge^{4} \mathcal{H}_{8}$.

\begin{tabular}{ccccccccc}
\hline \hline$\mu$ & $\kappa_{1}^{\mu}$ & $\kappa_{2}^{\mu}$ & $\kappa_{3}^{\mu}$ & $\kappa_{4}^{\mu}$ & $\kappa_{5}^{\mu}$ & $\kappa_{6}^{\mu}$ & $\kappa_{7}^{\mu}$ & $\kappa_{8}^{\mu}$ \\
\hline 1 & -1 & 0 & 0 & 1 & 0 & 1 & 1 & 0 \\
2 & -1 & 0 & 0 & 1 & 1 & 0 & 0 & 1 \\
3 & -1 & 0 & 1 & 0 & 0 & 1 & 0 & 1 \\
4 & -1 & 1 & 0 & 0 & 0 & 0 & 1 & 1 \\
5 & 0 & -1 & 0 & 1 & 0 & 1 & 0 & 1 \\
6 & 0 & 0 & -1 & 1 & 0 & 0 & 1 & 1 \\
7 & 0 & 0 & 0 & 0 & -1 & 1 & 1 & 1 \\
\hline \hline
\end{tabular}

TABLE VIII. Number of Slater determinants in the full and pinned CI expansions of the wave function for the spin-restricted system $\wedge{ }^{4} \mathcal{H}_{8}$ with $\mathbf{S}_{z}=1$.

\begin{tabular}{lccccc}
\hline \hline Condition & $|0\rangle$ & $\mathrm{S}$ & $\mathrm{D}$ & $\mathrm{T}$ & Total \\
\hline $\mathrm{CI}$ & 1 & 6 & 9 & 0 & 16 \\
$\mathbf{D}_{4,8}^{14}|\Psi\rangle=0$ & 1 & 0 & 9 & 0 & 10 \\
\hline \hline
\end{tabular}

occurs for a single-determinant wave function. For instance,

$$
\begin{aligned}
& D_{4,8}^{1}:=-n_{1}+n_{4}+n_{6}+n_{7}=2\left(1-n_{1}\right), \\
& D_{4,8}^{8}:=2-n_{2}-n_{3}-n_{5}+n_{8}=2 n_{8}, \\
& D_{4,8}^{14}:=2-n_{1}-n_{2}-n_{3}+n_{4}=2\left(1-n_{1}\right) .
\end{aligned}
$$

Chakraborty and Mazziotti [7] computed the occupation numbers for the ground state of some four-electron molecules for rank equal to twice the number of electrons, employing a STO-3G basis set. In this range of approximation, the two energetically lowest orbitals of $\mathrm{LiH}$ are completely occupied (therefore $D_{4,8}^{1}=0$ ) and the Shull-Löwdin functional guarantees that doubly excited determinants completely govern rank 8 CI calculations for this molecule.

However, there are important effects of dynamical electron correlation which involve the core electrons, and the molecule cannot be considered a two-electron system. In fact, for higher ranks the two biggest occupation numbers $\left(n_{1}=n_{2}\right)$ become smaller than 1. The first (and the second as well) occupation number of $\mathrm{BH}$ is very close to 1 , and accordingly $D_{4,8}^{1}$ is quasipinned. For $\mathrm{LiH}$ and $\mathrm{BeH}_{2}$, the seventh occupation number is almost 0 , and hence for these systems $D_{4,8}^{8}$ is quasipinned.

In a spin-compensated description, the system $\wedge^{4} \mathcal{H}_{8}$ with total spin component $\mathbf{S}_{z}$ equal to 1 contains 16 configurations, corresponding to $\wedge^{3} \mathcal{H}_{4} \otimes \mathcal{H}_{4}$. The CI expansion only contains double or single excitations. In a spin-uncompensated description, the system $\wedge^{4} \mathcal{H}_{8}$ with total spin component $\mathbf{S}_{z}$ equal to 1 would contain 30 configurations, corresponding to $\wedge^{3} \mathcal{H}_{5} \otimes \mathcal{H}_{3}$. Note that if the GPC

$$
D_{4,8}^{14}=2-n_{1}-n_{2}-n_{3}+n_{4} \geqslant 0
$$

is completely saturated, the corresponding wave function is a member of the 0-eigenspace of the operator:

$$
\mathbf{D}_{4,8}^{14}=\mathbf{2}-a_{1}^{\dagger} a_{1}-a_{2}^{\dagger} a_{2}-a_{3}^{\dagger} a_{3}+a_{4}^{\dagger} a_{4} ;
$$

and for both configurations, single and triple excitations are entirely suppressed. This is a nontrivial fact. See Tables VIII and IX. Besides the initial configuration,

$$
\left|\alpha_{1} \alpha_{2} \alpha_{3} \alpha_{4}\right\rangle
$$

TABLE IX. Number of Slater determinants in the full and pinned $\mathrm{CI}$ expansions of the wave function for the spin-unrestricted system $\wedge{ }^{4} \mathcal{H}_{8}$ with $\mathbf{S}_{z}=1$.

\begin{tabular}{lccccc}
\hline \hline Condition & $|0\rangle$ & $\mathrm{S}$ & $\mathrm{D}$ & $\mathrm{T}$ & Total \\
\hline CI & 1 & 8 & 16 & 5 & 30 \\
$\mathbf{D}_{4,8}^{14}|\Psi\rangle=0$ & 1 & 0 & 11 & 0 & 12 \\
\hline \hline
\end{tabular}


the configurations present in the expansion are just double excitations of this state, which, in addition, do not contain the fourth natural orbital $\alpha_{4}$.

In general, for the system $\wedge^{N} \mathcal{H}_{m}$, the condition

$$
(N-2)+n_{N}=n_{1}+\ldots+n_{N-1}
$$

has as a consequence that only double excitations become the relevant configurations in a CI expansion [38]. Moreover, the only configuration containing the orbital $\alpha_{N}$ is $\left|\alpha_{1} \alpha_{2} \cdots \alpha_{N}\right\rangle$.

For the larger system $\wedge^{4} \mathcal{H}_{10}$, the occupation numbers are bounded by 121 constraints $[4,33]$. We postpone their study.

\section{CONCLUSION}

The recent solution of the pure $N$-representability problem, due to Klyachko, promises to generate a wide set of conditions (the GPCs) on the natural occupation numbers for fermionic systems. The Klyachko algorithm does indeed produce sets of linear inequalities with integer coefficients for those numbers. The derivation of these inequalities, and of their consequences, is still a work in progress.

For reasons that nobody has been quite able to fathom yet, some of these inequalities appear to be nearly saturated, in a far from random way: this is the quasipinning phenomenon. A research program is born around these facts.

By means of both theoretical and numerical results, in this paper we have continued to explore the nature of pinning and quasipinning in some atomic and molecular models (mainly perturbed lithium with broken spherical symmetry and the dimer ion $\mathrm{He}_{2}^{+}$), for several finite-rank approximations whose GPCs are known.

We sum up our opinions on the outcomes of that program, so far.

(a) Saturation of some of the GPCs leads to strong selection rules for identifying the most (in)effective configurations in CI expansions. In simple cases, this provides means for reducing the number of Slater determinants in the CI picture and therefore reducing the computational requirements $[5,6,13]$. In general, it does provide insights into the structure of the wave function, which brute force methods are unable to do. (b) However, it is unlikely that the Klyachko paradigm will be relevant for computational quantum chemistry, at least in the short run. The main problem is the dramatic increase in the number of GPCs with the rank of the spin orbital systems introduced in the calculations.

(c) The robustness of the near-saturation of a particular type of constraint conspires to "explain" why double excitations govern CI calculations of electron correlation, when using natural orbitals [38].

(d) A natural question is whether the exact "Löwdin-Shull" formula, (6), for three-electron systems can be generalized to a higher rank. The answer is a qualified, approximate yes, the price to pay being to invoke a second type of constraint less strongly quasipinned than the one referred to in point (c). We refrain from going into the details here.

(e) A very promising avenue of research is to use the GPCs to improve on the 1-RDM theory. There are now quite a few physically motivated density matrix functionals in the literature, built from the knowledge of the natural orbitals and occupation numbers, which can be traced back to the one proposed by Müller 30 years ago [39]; they have mostly amounted to figuring out ansatze for reasonable twobody reduced density matrices, failing, to date, to fulfill one physical requirement or another [40]. The approach discussed in this paper suggests constructing a 1-RDM by restricting the minimization set to the subset of GPC-honest systems. A promising start in this direction is [9].

\section{ACKNOWLEDGMENTS}

The authors are most grateful to E. R. Davidson, J. M. Gracia-Bondía, D. Gross, R. Herrero-Hahn, S. Kohaut, M. Marques, D. A. Mazziotti, C. Schilling, J. C. Várilly, and M. Walter for helpful comments and illuminating discussions. We thank N. Louis for IT support. C.L.B.R. was supported by the Departamento Administrativo de Ciencia, Tecnología e Innovación (Colciencias) under a Francisco José de Caldas scholarship. He very much appreciates the warm atmosphere of the Physikalische und Theoretische Chemie group at Universität des Saarlandes.
[1] A. J. Coleman, Rev. Mod. Phys. 35, 668 (1963).

[2] R. E. Borland and K. Dennis, J. Phys. B 5, 7 (1972).

[3] A. Klyachko, J. Phys.: Conf. Ser. 36, 72 (2006).

[4] M. Altunbulak and A. Klyachko, Commun. Math. Phys. 282, 287 (2008)

[5] C. Schilling, D. Gross, and M. Christandl, Phys. Rev. Lett. 110, 040404 (2013).

[6] C. L. Benavides-Riveros, J. M. Gracia-Bondía, and M. Springborg, Phys. Rev. A 88, 022508 (2013).

[7] R. Chakraborty and D. A. Mazziotti, Phys. Rev. A 89, 042505 (2014).

[8] C. L. Benavides-Riveros and C. Schilling (unpublished) (2015).

[9] I. Theophilou, N. N. Lathiotakis, M. A. L. Marques, and N. Helbig, J. Chem. Phys. 142, 154108 (2015).

[10] R. Chakraborty and D. A. Mazziotti, Phys. Rev. A 91, 010101(R) (2015).
[11] J. Ivanic and K. Ruedenberg, Theor. Chem. Acc. 106, 339 (2001).

[12] E. Giner, A. Scemama, and M. Caffarel, Can. J. Chem. 91, 879 (2013).

[13] A. Klyachko, arXiv:0904.2009.

[14] C. Schilling, Phys. Rev. A 91, 022105 (2015).

[15] W. B. McRae and E. R. Davidson, J. Math. Phys. 13, 1527 (1972).

[16] E. R. Davidson, Comp. Theor. Chem. 1003, 28 (2013).

[17] R. Pauncz, Spin Eigenfunctions: Construction and Use (Plenum Press, New York, 1979).

[18] C. Schilling in Mathematical Results in Quantum Mechanics 12 (World Scientific, Singapore, 2014).

[19] M. Walter, Ph.D. thesis, ETH, Zürich (2014), arXiv:1410.6820.

[20] A. Lopes, D. Gross, and C. Schilling (unpublished) (2015).

[21] C. Schilling, arXiv:1506.08833. 
[22] P.-O. Löwdin and H. Shull, Phys. Rev. 101, 1730 (1956).

[23] C. Schilling pointed out to us at once that there is no ambiguity of signs in display (6).

[24] W. Kutzelnigg and V. H. Smith, Int. J. Quant. Chem. 2, 531 (1968).

[25] P. Ziesche, Phys. Lett. A 195, 213 (1994).

[26] D. A. Mazziotti, Phys. Rev. Lett. 108, 263002 (2012).

[27] D. A. Mazziotti, Phys. Rev. A 85, 062507 (2012).

[28] N. N. Lebedev, Special Functions and Their Applications (Dover, Mineola, NY, 1972).

[29] Wolfram Research, Mathematica, Version 10.0 (Wolfram Research, Champaign, IL, 2014).

[30] R. D. Johnson, Computational Chemistry Comparison and Benchmark Database, Tech. Rep. 101 Release $16 a$ (NIST, Gaithersburg, MD, 2013).
[31] J. Xie, B. Poirier, and G. I. Gellene, J. Chem. Phys. 122, 184310 (2005).

[32] W. J. Hehre, R. Ditchfield, and J. A. Pople, J. Chem. Phys. 56, 2257 (1972).

[33] See, Supplemental Material in Ref. [4].

[34] A. E. Nilsson and S. Johansson, Phys. Scripta 44, 226 (1991).

[35] C. L. Benavides-Riveros, J. M. Gracia-Bondía, and J. C. Várilly, Phys. Rev. A 86, 022525 (2012).

[36] Ł. M. Mentel, R. van Meer, O. V. Gritsenko, and E. J. Baerends, J. Chem. Phys. 140, 214105 (2014).

[37] D. W. Smith, Phys. Rev. 147, 896 (1966).

[38] C. L. Benavides-Riveros, J. M. Gracia-Bondía, and M. Springborg, arXiv:1409.6435.

[39] A. M. K. Müller, Phys. Lett. A 105, 446 (1984).

[40] C. L. Benavides-Riveros and J. C. Várilly, Eur. Phys. J. D 66, 274 (2012). 